\title{
Characterization of Small Areas of Thin-Films by Grazing-Exit Electron Probe X-ray Microanalysis *1
}

\author{
Kouichi Tsuji ${ }^{* 2}$, Zoya Spolnik, Kazuaki Wagatsuma, Kesami Saito and \\ Katsuhiko Asami \\ Institute for Materials Research, Tohoku University, Sendai 980-8577, Japan
}

\begin{abstract}
Metallic thin films deposited on Si wafers were analyzed by EPMA under grazing-exit geometry. The experimental setup consisted of the conventional SEM and EDX. The exit angle was controlled by moving the EDX up and down. After the SEM observation, the electron beam fixed on the analyzed position. And then, the intensities of characteristic X-rays were measured as a function of exit angle. These angle dependences were analyzed by curve fitting of the simulated curves. As a result, the thickness and the density of thin films were evaluated. The difference of the density of chromium thin films prepared by different methods was found. The GE-EPMA measurement was performed for $\mathrm{Au}-\mathrm{Ag}$ layers on the $\mathrm{Si}$ wafer. The thin-film characterization for each layer was independently performed at localized region on $\mathrm{Ag}$ and $\mathrm{Au}$ layers.
\end{abstract}

(Received October 1, 2001; Accepted November 21, 2001)

Keywords: thin film analysis, electron probe microanalysis, silicon wafer, gold film

\section{Introduction}

Thin-films play an important role in semiconductor devices. The evaluation and control of elemental composition, thickness and density of the films are necessary to obtain the optimum functions of semiconductor devices. The evaluation of thin-film thickness of nano-meter order is possible by X-ray analytical method. For example, thickness is evaluated by analyzing the oscillation structure observed in the angle-dependence of the X-ray reflectivity, as shown in Fig. 1(a). However, the information obtained from such measurements is the averaged one on the whole surface of the sample, because the X-rays irradiate the whole sample at the small angle of incidence. In addition, the sample should be flat for grazing-incidence measurements. Since the size of the semiconductor devices is decreased more and more, the analytical method to evaluate thin-films at small region is needed.

Recently, we have proposed a new analytical method, grazing-exit electron prove microanalysis (GE-EPMA). ${ }^{1-3)}$ In the conventional EPMA, the characteristic X-rays are measured at large take-off angle, approximately $40^{\circ}$. In the case of GE-EPMA, the X-rays are measured at extremely small angle (grazing-exit angle), normally less than $1^{\circ}$. This experimental configuration makes surface analysis possible. Due to refraction effect on the surface, ${ }^{4)}$ the X-rays emitted from the deep inside of the sample are cut by the slit, which is placed between the sample and the X-ray detector; as a result, only the X-rays emitted from the surface of the sample can be detected. In the GE-EPMA, electron beam irradiates the sample at a right angle; therefore, the localized X-ray analysis is possible, as shown in Fig. 1(b). In this method, the sample is not requested to have flatness on the whole surface.

In this paper, we demonstrate the localized thin-film analysis by GE-EPMA method. For this purpose, we have developed the curve-fitting method using the calculated

${ }^{* 1}$ This Paper was Presented at the Autumn Meeting of the Japan Institute of Metals, held in Fukuoka, on September 22, 2001.

${ }^{* 2}$ Corresponding author: E-mail: tsuji@imr.tohoku.ac.jp (a) Graizng-incidence X-ray analysis

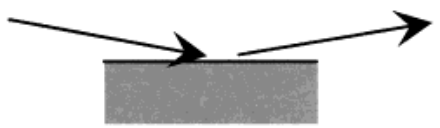

(b) Graizng-exit X-ray analysis

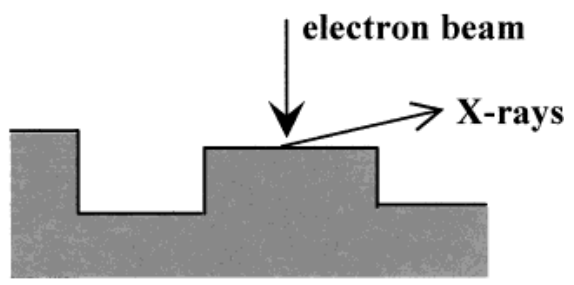

Fig. 1 (a) Grazing-incidence X-ray analysis. (b) Grazing-exit X-ray analysis.

angle-intensity curve for the characteristic X-rays.

\section{Experimental}

Figure 2 shows the schematic drawing of the experimental setup used for GE-EPMA. The details of the GE-EPMA setup has been described elsewhere. ${ }^{5)}$ The SEM (JSM-5500, Jeol, Japan) and the EDX (energy-dispersive X-ray detector, Horiba, Japan) have been combined by a stain-less steel flexible tube. The electron beam (accelerating voltage: $20 \mathrm{kV}$, beam current: $0.5 \mathrm{nA}$ ) irradiated the sample at an incident angle of $90^{\circ}$. To change the exit angle of the characteristic $\mathrm{X}$-rays, the EDX was moved up and down by using a linear movement Z-stage driven by a stepping motor, as shown in Fig. 2. A computer controlled both the EDX analysis and the Z-stage; therefore, the automatic measurement of the angle-dependence of the X-ray intensity was possible. 


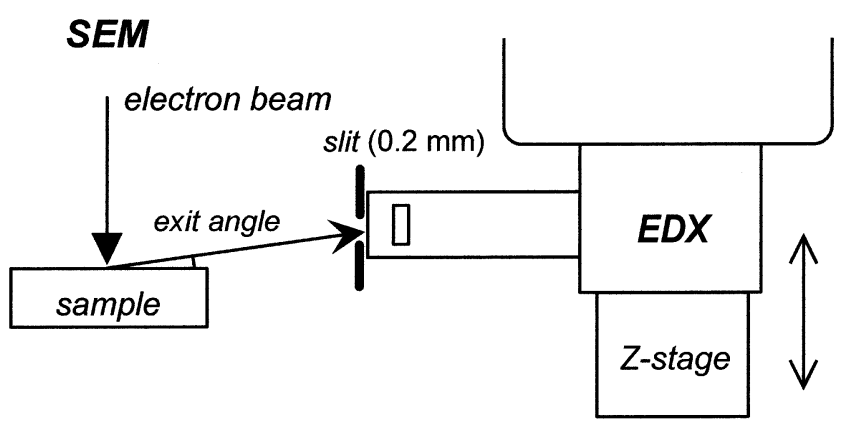

Fig. 2 Schematic drawing of SEM-EDX system used for GE-EPMA measurements.

\section{Calculation of Electron Induced X-ray Intensity at Grazing-Exit Angle}

The electron-induced X-ray intensity was calculated as a function of exit angle to analyze the GE-EPMA result. The calculation procedure includes two processes. The first process is the X-ray production by electron irradiation. The X-ray production distribution $I_{e}(z)$ in depth $(z)$ can be obtained from Monte-Carlo simulation. ${ }^{6)}$ The second process is the X-ray emission at the grazing-exit angle. A multi-layered model was considered to analyze the thin films. ${ }^{7)}$ The X-ray intensity emitted from the analyzed layer was calculated as a function of exit angle by considering the reflection and refraction on each interface. By combining calculations of two processes, the characteristic X-ray intensity induced by electron irradiation was obtained as a function of exit angle.

\section{Results and Discussions}

First, the calculated X-ray intensity was compared with the experimental result obtained for a simple sample, that is, a $\mathrm{Si}$ wafer. In Fig. 3, the measured $\mathrm{Si} \mathrm{K} \alpha$ intensities are plotted with circles as a function of exit angle. At each exit angle, the X-rays were measured for approximately $300 \mathrm{~s}$, depending on the sample. Totally, several hours were taken. $\mathrm{Si} \mathrm{K} \alpha$ increased at an exit angle of about $1^{\circ}$, which corresponds to the critical angle for total reflection. The simulated curve described with solid line agreed well with the experimental plots. This suggests that the calculation procedure of the X-ray intensity is right.

Two types of $\mathrm{Cr}$ thin films were measured. One $\mathrm{Cr}$ film was prepared by a magnetron sputtering method at Laboratory for Advanced Materials (LAM) of Tohoku University. Another $\mathrm{Cr}$ film was prepared by a vacuum evaporation method. The experimental results for two samples are shown in Fig. 4. The $\mathrm{Cr} \mathrm{K} \alpha$ intensity for the $\mathrm{Cr}$ film prepared by the magnetron sputtering increased at larger exit angle compared with that for the $\mathrm{Cr}$ film prepared by the vacuum evaporation. The critical angle, where the characteristic X-ray intensity increases, depends on the density of the surface of the materials. ${ }^{8)}$ Therefore, this difference of the angle dependency indicates the difference of the densities of both $\mathrm{Cr}$ films. The experimental plots were fitted with the curves calculated with different densities for $\mathrm{Cr}$ thin film. The best curve fitting for magnetron sputtering was obtained with the Cr bulk density $\left(7.2 \mathrm{~kg} / \mathrm{m}^{3}\right)$ and the thickness of $180 \mathrm{~nm}$. However, the best

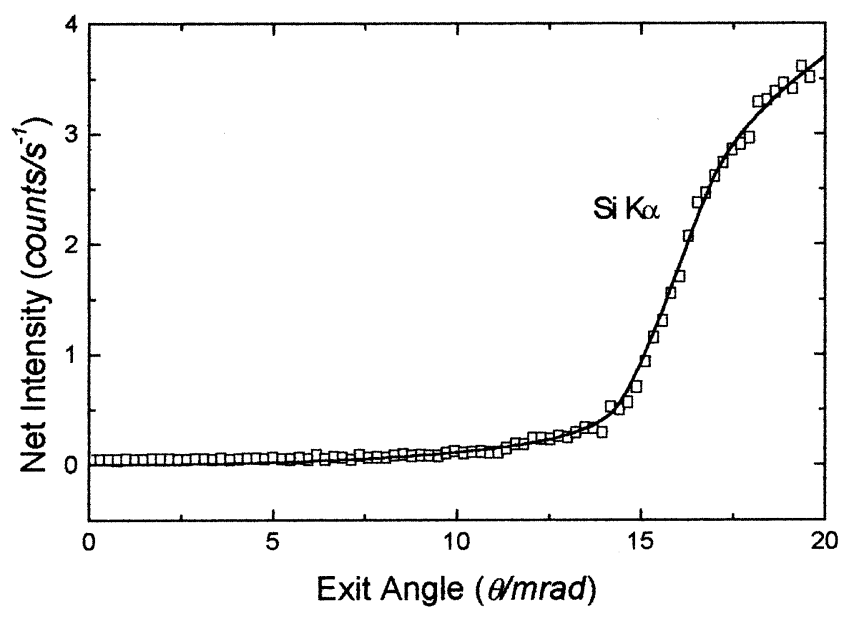

Fig. 3 Si K $\alpha$ intensity plotted for Si substrate as a function of exit angle. The calculate curve is also shown with solid line.

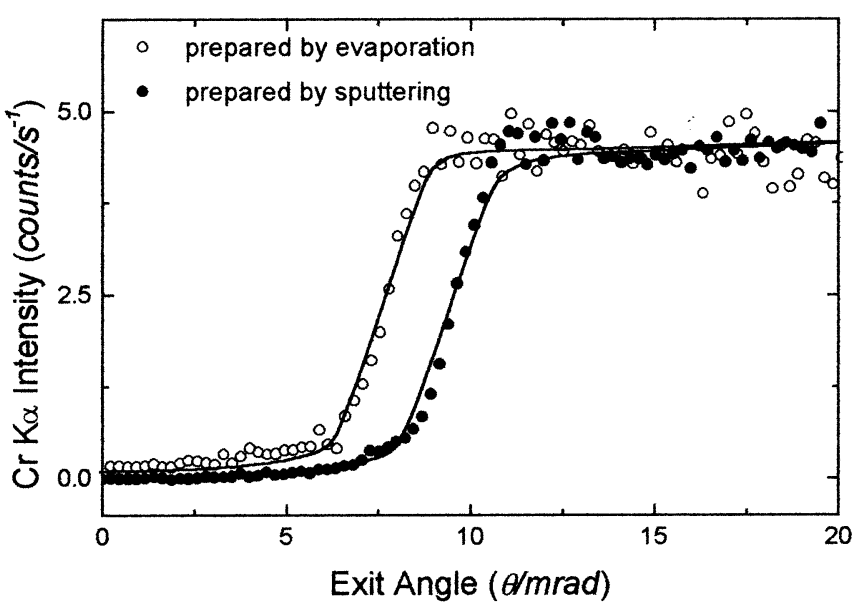

Fig. $4 \mathrm{Cr} \mathrm{K} \alpha$ intensity plotted as a function of exit angle, measured for $\mathrm{Cr}$ thin films prepared by magnetron sputtering and vacuum evaporation. The calculated curves are also shown with solid lines.

fitting for vacuum evaporation was obtained with the reduced density of $4.8 \mathrm{~kg} / \mathrm{m}^{3}$ and the thickness of $200 \mathrm{~nm}$. This is probably that the $\mathrm{Cr}$ film prepared by the vacuum evaporation has a porous structure and include light chemical species such as organic materials, which would produced in the vacuum chamber. Although the reason of the reduction of the density is not clear, it is found that the GE-EPMA measurement is useful for thin-film characterization, that is the evaluation of the thickness and density of thin films.

Next, more complicated sample was measured. As shown in the inset of Fig. 5(a), Ag and Au thin films were deposited on the Si substrate by vacuum evaporation method in the dimension of $2 \times 10 \mathrm{~mm}$. The thickness and the density of each film were evaluated by GE-EPMA method. From SEM observation, the analyzed position was determined on the $\mathrm{Ag}$ thin film. After the electron beam was fixed on this position, the angle dependence of $\mathrm{Ag} \mathrm{L} \alpha$ intensity was measured. The result is shown in Fig. 5(a). The theoretical curve was tried to fit to the experimental plots. The best fitting was obtained with a thickness of $40 \mathrm{~nm}$ and a density of $8.0 \mathrm{~kg} / \mathrm{m}^{3}$, as shown in Fig. 5(a). The obtained density was smaller than Ag bulk density. Next, for the same sample, the same angle-dependent measurement was performed on the Au layer, as shown in 


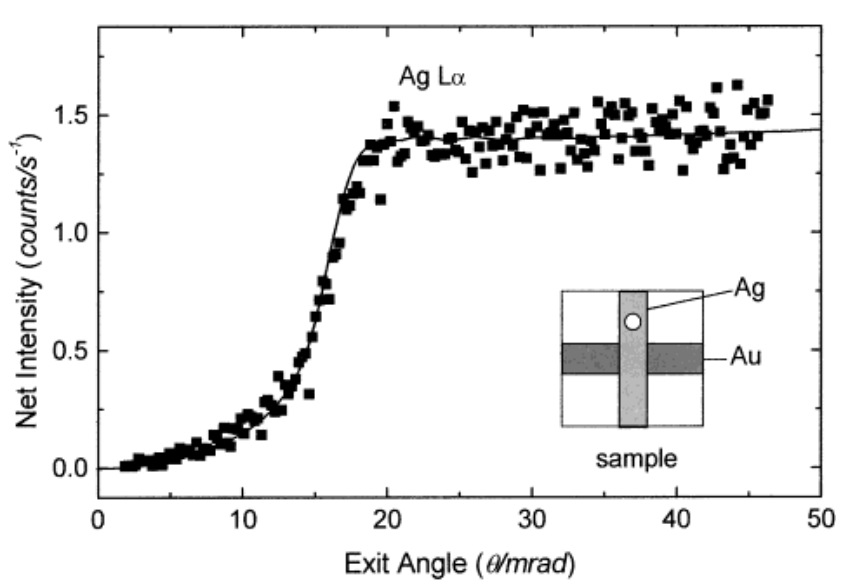

(a)

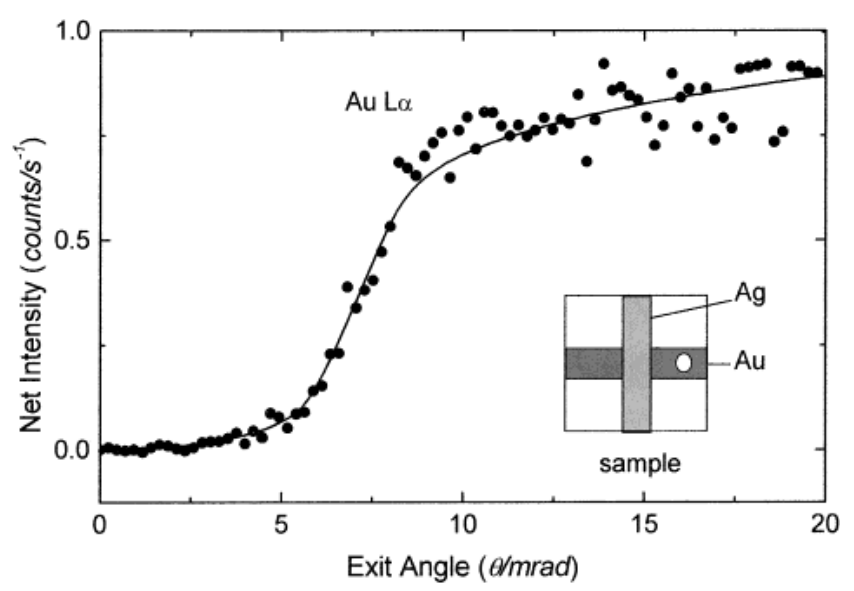

(b)

Fig. 5 Localized thin-film analysis for $\mathrm{Ag}-\mathrm{Au}$ layers deposited on $\mathrm{Si}$ substrate. Experimental plots for $\operatorname{Ag} \operatorname{L} \alpha$ (a) and $\mathrm{Au} \operatorname{L} \alpha$ (b), which were independently measured at localized regions marked with white circles in each inset, are shown with the best fitting of calculated curves.

the inset of Fig. 5(b). Compared with the experimental plots for the Ag film, Au L $\alpha$ intensity increased at smaller exit angle, approximately $7 \mathrm{mrad}$. This is due to the difference of wavelength of $\mathrm{Ag} \mathrm{L} \alpha$ and $\mathrm{Au} \mathrm{L} \alpha$. (The critical angle for total reflection depends on the wavelength of X-rays. ${ }^{8)}$ ) The best fit of the theoretical curve was obtained with a thickness of $80 \mathrm{~nm}$ and a density of $15.0 \mathrm{~kg} / \mathrm{m}^{3}$, as shown in Fig. 5(b). Here, we emphasize that this thin-film characterization could be possible for localized region, depending on the diameter of electron beam. That is to say, localized thin-film analysis is possible by GE-EPMA. It would be impossible to characterize independently each thin film of the sample of Fig. 5 by glazing-incidence X-ray analytical method, such as X-ray reflectivity measurement.

\section{Conclusions}

The metallic thin films were analyzed by EPMA under grazing-exit geometry. For this measurement, the conven- tional SEM and the EDX were combined. Usually, the exit angle is changed by tilting the sample. In our new GE-EPMA setup, the exit angle is changed by moving the EDX. This is suitable for localized analysis, because the analyzed position is stable even if the exit angle is changed. ${ }^{9)}$ Thin films were analyzed by curve fitting method, that is, the simulated curves were compared with the angle-dependent experimental data. The thickness and the density of thin films were evaluated. For the $\mathrm{Cr}$ thin films prepared by the different methods, the difference of the density was found for these two samples. The density of the $\mathrm{Cr}$ film prepared by the magnetron sputtering was almost the bulk (Cr) density, however, the density of the $\mathrm{Cr}$ film prepared by the vacuum evaporation was about $67 \%$ of the bulk density. The GE-EPMA measurement was performed for $\mathrm{Au}-\mathrm{Ag}$ layers on the Si wafer. After the SEM observation, the analyzed position was decided. The thin-film characterization for each layer was independently performed at localized region on $\mathrm{Ag}$ and Au layers. The determined values (thickness and density) would include the error of $\pm 10 \%$. The accuracy will be improved by applying stronger electron beam current, which increases the intensity of the characteristic X-rays. Also, the multi-detection system will be useful for GE-EPMA measurement. That is, if the characteristic X-rays are detected by many X-ray detectors from the various directions under grazing-exit conditions, stronger X-rays can be detected for the short time, leading to the improvement of the accuracy and the decrease of the measuring time.

\section{Acknowledgements}

One of the authors (K. Tsuji) was financially supported by Grant-in-Aid (B-12554030) from the Ministry of Education, Science, Sports and Culture Japan, and by the grant of Shimadzu Science Foundation. Z. Spolnik was also financially supported by the Japan Society for Promotion of Science (JSPS, No. L00548). This work was performed under the research program (\#234) at Laboratory for Advanced Materials (LAM) of Institute for Materials Research (IMR), Tohoku University.

\section{REFERENCES}

1) K. Tsuji, K. Wagatsuma, R. Nullens and R. Van Grieken: Anal. Chem. 71 (1999) 2497-2501.

2) K. Tsuji, Z. Spolnik, K. Wagatsuma, R. Nullens and R. Van Grieken: Mikrochim. Acta 132 (2000) 357-360.

3) K. Tsuji: Materia 39 (2000) 586-593 (in Japanese).

4) S. Hasegawa, S. Ino, Y. Yamamoto and H. Daimon: Jpn. J. Appl. Phys. 24 (1985) L387-390.

5) K. Tsuji, Z. Spolnik and T. Ashino: Rev. Sci. Instrum. 72 (2001) 39333936.

6) V. D. Scott, G. Love and S. J. B. Reed: Quantitative Electron-Probe Microanalysis, (Ellis Horwood, New York, 1995) pp. 212-233.

7) K. Tsuji and K. Hirokawa: J. Appl. Phys. 75 (1994) 7189-7194.

8) R. Klockenkamper: Total-Reflection X-ray Fluorescence Analysis, (John Wiley and Sons, New York, 1997) pp. 29-38.

9) K. Tsuji, Z. Spolnik, K. Wagatsuma, S. Nagata and I. Satoh: Anal. Sci. 17 (2001) 145-148. 